\title{
Doppler Effect on Nanopatterning with Nonlinear Laser Lithography
}

\author{
Ö. Yavuz ${ }^{1}$, Semih Kara ${ }^{1}$, O. Tokel ${ }^{2}$, I. Pavlov ${ }^{2}$ and F. Ö. Ilday ${ }^{1,2}$ \\ ${ }^{1}$ Department of Electrical and Electronics Engineering, Bilkent University, 06800 Ankara, Turkey \\ ${ }^{2}$ Department of Physics, Bilkent University, 06800 Ankara, Turkey
}

Just five years after invention of the laser, laser induced periodic structures (LIPSS) had been reported [1]. However, the structure period is not very uniform in LIPSS. Recently, with nonlinear laser lithography (NLL), long range ordered periodic surface structures had been maintained by exploiting various feedback mechanisms and nonlinearities [2]. Albeit, fine tuning of structure period remains challenging. Here, we present an analogy between doppler effect and structure period of the NLL which adds a capability of changing the structure period.

In NLL, assuming that the surface profile consists of a point defect and incidence is occurred with an angle of $\varphi$, period of the structures can be written as

$$
\Lambda^{\prime}=\frac{\Lambda}{1-\sin \varphi \cos \theta}
$$

, where $\theta$ is polarisation angle and $\Lambda$ is structure period with normal incidence, $\Lambda^{\prime}$ is the new period. Note that, the equation is as same as Doppler effect with moving source and still observer with a replacement of speed of the source with $c \sin \varphi$ ignoring the relativistic effects.

Simulations has been done according to the enhanced version of the theory which is written in Ref. 2. Period of the structures is considered as $870 \mathrm{~nm}$ which is taken from experiments on $50 \mathrm{~nm}$ thick titanium sample.

In order to compare our work with Doppler shift, Doppler shift for moving source and still observer scheme is explained in Fig. 1 (a). Total intensity profile created on the surface by a single, point-like defect sitting at the centre $(\mathrm{x}=0, \mathrm{y}=0)$ can be seen in Fig. 1 (b). Doppler equation is simulated considering Eqn. (1), where $\theta$ is polarisation angle and $\varphi$ is incidence (tilt) angle. From Fig. 1 (c) and (d), it can be seen that simulation results agrees very well with the expected values from Doppler shift equations.
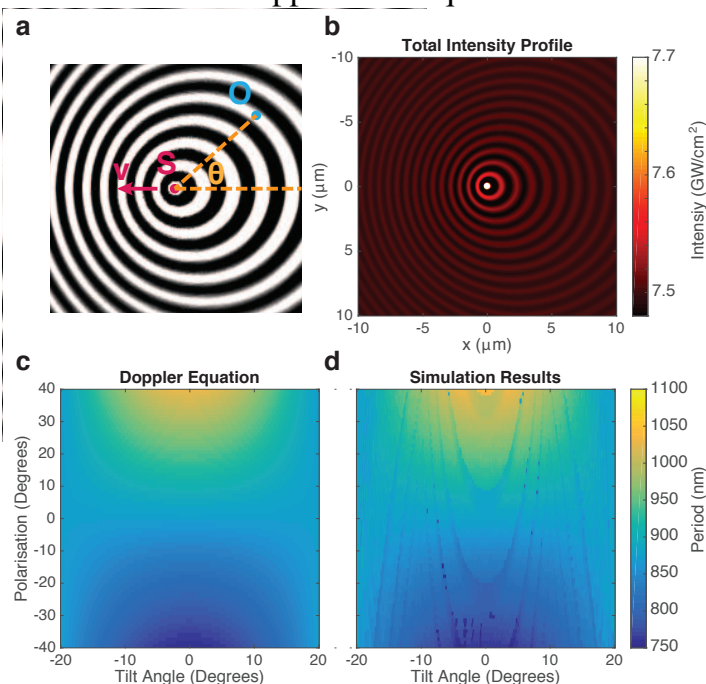

d

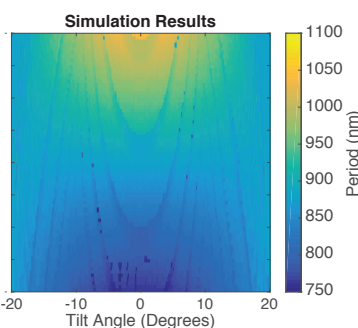

Fig. 1 (a) Schematic of Doppler shift with moving source where $S$ is position of source, $O$ is position of observer, v is speed of source, and $\theta$ is angle defined in between source and observer. (b) Simulation results of total intensity profile from single, point like structure sitting at the centre of simulation domain. (c) Expected period of the structures according to Doppler effect. (d) Structure period obtained from the simulation.

In experiments, $200 \mathrm{fs}$ pulse duration, $1 \mathrm{MHz}$ repetition rate, $\lambda=1030 \mathrm{~nm}$ laser has been used. In preliminary experiments angle of polarisation is always 0 degrees, and period reduces to $\frac{\Lambda}{1 \pm \sin \varphi}$. According to experiments, for incidence angle of 15 degrees, scanning along one direction (towards shorter period) creates structures with 700 $\mathrm{nm}$ period, and scanning along the inverse direction (towards longer period) creates structures with $1.1 \mu \mathrm{m}$ period. The initial experiments show great agreement with the simulation results.

In conclusion, here, we present a analogy between Doppler effect and Nonlinear Laser Lithography. The initial experiments shows great agreement with the simulations and further experiments are in progress. This is the first time that the Doppler effect is shown in material processing. Using the analogy, structures with various periods can be fine tuned and produced.

\section{References}

[1] M. Birnbaum, "Semiconductor surface damage produced by ruby lasers," J. Appl. Phys. 36, 3688 (1965).

[2] B. Öktem, I. Pavlov, S. llday, H. Kalaycıŏlu, A. Rybak, S. Yavaş, M. Erdoğan, and F. Ö. llday, "Nonlinear laser lithography for indefinitely large-area nanostructuring with femtosecond pulses," Nature Photon. 7, 897 (2013). 